\title{
The Powerful Jet and Gamma-Ray Flare of the Quasar PKS 0438-436
}

\author{
Brian Punsly ${ }^{1}$, Andrea Tramacere ${ }^{2}$, Preeti Kharb ${ }^{3}$ and Paola Marziani ${ }^{4}$
}

\begin{abstract}
PKS 0438-436 at a redshift of $z=2.856$ has been previously recognized as possessing perhaps the most luminous known synchrotron jet. Little is known about this source since the maximum elevation above the horizon is low for the Very Large Array (VLA). We present the first VLA radio image that detects the radio lobes. We use both the $151 \mathrm{MHz}$ luminosity, as a surrogate for the isotropic radio lobe luminosity, and the lobe flux density from the radio image to estimate a long term, time averaged, jet power, $\bar{Q}=1.5 \pm 0.7 \times 10^{47} \mathrm{ergs} \mathrm{s}^{-1}$. We analyze two deep optical spectra with strong broad emission lines and estimate the thermal bolometric luminosity of the accretion flow, $L_{\mathrm{bol}}=6.7 \pm 3.0 \times 10^{46} \mathrm{ergs} \mathrm{s}^{-1}$. The ratio, $\bar{Q} / L_{\mathrm{bol}}=3.3 \pm 2.6$, is at the limit of this empirical metric of jet dominance seen in radio loud quasars and this is the most luminous accretion flow to have this limiting behavior. Despite being a very luminous blazar, it previously had no $\gamma$-ray detections (EGRET, AGILE or FERMI) until December 11 - 132016 (54 hours) when FERMI detected a flare that we analyze here. The isotropic apparent luminosity from $100 \mathrm{MeV}$ - $100 \mathrm{GeV}$ rivals the most luminous detected blazar flares (averaged over 18 hours), $\sim 5-6 \times 10^{49} \mathrm{ergs} \mathrm{s}^{-1}$. The $\gamma$-ray luminosity varies over time by two orders of magnitude, highlighting the extreme role of Doppler abberation and geometric alignment in producing the inverse Compton emission.
\end{abstract}

Subject headings: black hole physics — galaxies: jets - galaxies: active - accretion, accretion disks

\footnotetext{
${ }^{1} 1415$ Granvia Altamira, Palos Verdes Estates CA, USA 90274: ICRANet, Piazza della Repubblica 10 Pescara 65100, Italy and ICRA, Physics Department, University La Sapienza, Roma, Italy, brian.punsly@cox.net

${ }^{2}$ Department of Astronomy, University of Geneva, Chemin d'Ecogia 16 - 1290 - Versoix - Switzerland

${ }^{3}$ National Centre for Radio Astrophysics, Tata Institute of Fundamental Research, Post Bag 3, Ganeshkhind, Pune 411007, India

${ }^{4} \mathrm{INAF}$, Osservatorio Astronomico di Padova, Italia
} 


\section{Introduction}

The quasar PKS 0438-436 at $\mathrm{z}=2.856$ has been identified as the radio source with the highest radio luminosity and the second strongest known synchrotron core (Morton et al. 1978; Punsly 1995). PKS 0438-436 is considered a blazar due to a flat spectrum (defined in terms of the flux density as $F_{\nu} \sim \nu^{-\alpha}$ ) with $\alpha<0.5$ and its optical polarization (Tingay et al. 2003; Impey and Tapia 1990). Not only are there epochs of high polarization, but it is highly variable, changing from $4.7 \%$ to $1.7 \%$ with a 150 degree swing in position angle in 7 months (Impey and Tapia 1990; Fugmann and Meisenheimer 1988). However, what is unusual is the enormous low frequency flux density compared to the most luminous blazars (Punsly 1995). The $160 \mathrm{MHz}$ flux density is $7.9 \mathrm{Jy}$ at $\mathrm{z}=2.856$ (Slee 1995). For comparison, we compute the $151 \mathrm{MHz}$ luminosity $L_{151} \approx 4.5 \times 10^{28} \mathrm{WHz}^{-1} \mathrm{sr}^{-1}$. This is the very rare object that lies near the high end of the luminosity distribution at both low frequency and high frequency ( $\geq 5 \mathrm{GHz}$ ) (Morton et al. 1978; Punsly 1995; Willott et al. 1999).

There are two unusual aspects of this luminous blazar, the large $L_{151}$ and the lack of a detection in the gamma rays, until now. A large $L_{151}$ is typically indicative of luminous radio lobes in which energy is stored (Rawlings and Saunders 1991). This study has three major objectives. First, determine the radio lobe morphology and flux density so we can estimate the long term time averaged radio jet power, $\bar{Q}$ (Section 2). Then, analyze the optical spectrum to get an estimate of the thermal bolometric luminosity of the accretion flow, $L_{\text {bol }}$ (Section 3). In Section 4, we analyze the December 2016 gamma ray flare. In this paper we adopt the following cosmological parameters: $H_{0}=70 \mathrm{~km} \mathrm{~s}^{-1} \mathrm{Mpc}^{-1}, \Omega_{\Lambda}=0.7$ and $\Omega_{m}=0.3$.

\section{The Large Scale Radio Structure and the Jet Power}

Estimates of instantaneous blazar jet power are often based on radio core and $\gamma$-ray emission (eg. the methods of Ghisellini et al. (2010)) and the estimated Doppler enhancement factor, $\mathcal{D}$, can introduce very significant uncertainty (Lind and Blandford 1985; Punsly and Tingay 2005). Estimating $\bar{Q}$ from the lobe luminosity is not instantaneous power. Yet, it has the virtue that the lobe luminosity and therefore $\bar{Q}$ is not sensitive to the large uncertainty of $\mathcal{D}$ (Rawlings and Saunders 1991; Willott et al. 1999). 

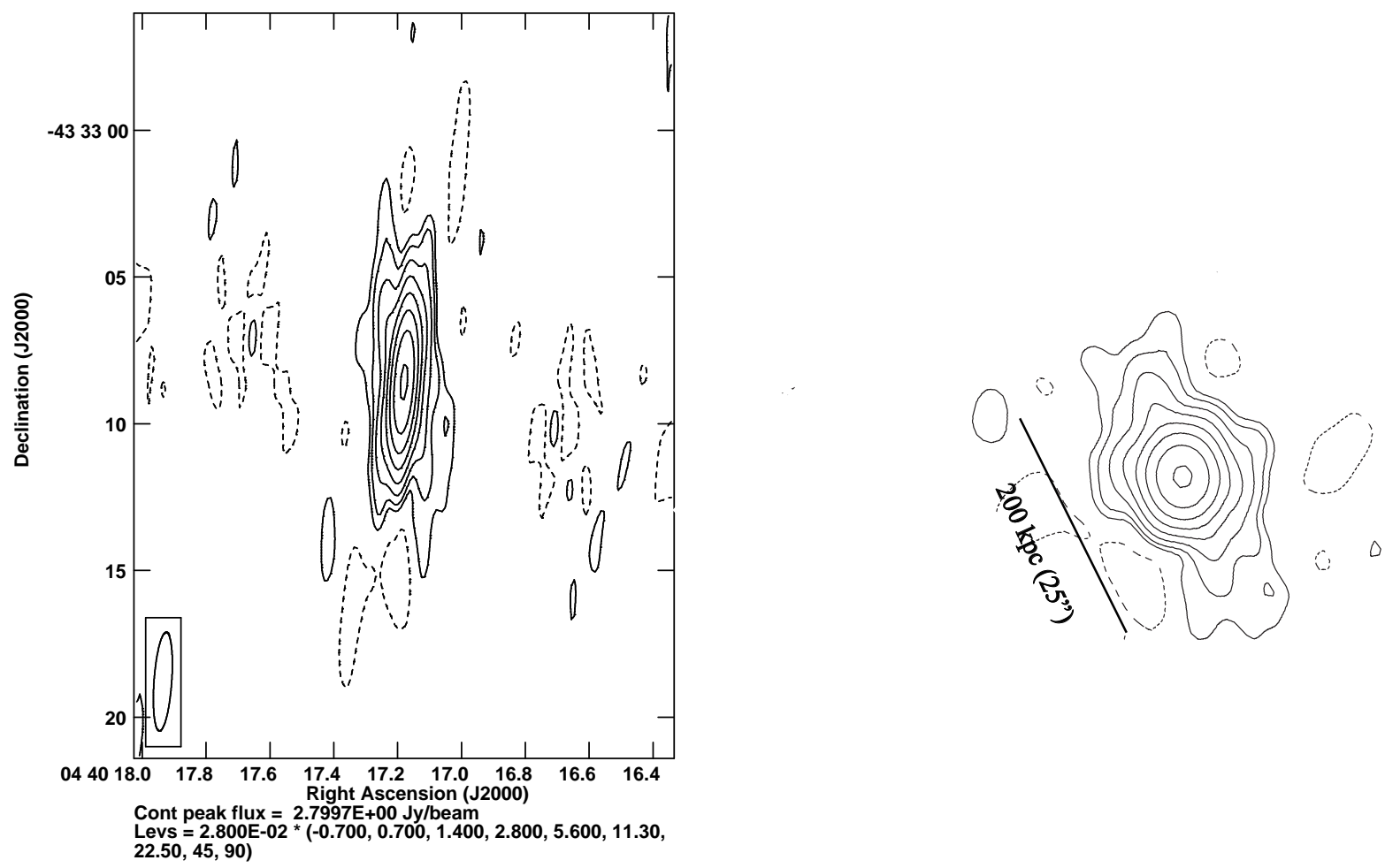

Fig. 1. - The left panel is the JVLA image at $2.5 \mathrm{GHz}$. The image is difficult to interpret, initially, due to the elongated beam shape and the compact east-west morphology. The right panel is a qualitative depiction of the intrinsic symmetry of the radio source that is designed to clarify the intrinsic morphology of the left hand image. It is an attempt to correct for a line of sight near the jet axis and a highly elongated beam. It assumes that the line of sight to the symmetry axis is $\psi=15^{\circ}$, which is used to simulate a rotation of the radio source into the sky plane. More details are provided in the text on the method and somewhat arbitrary choice for $\psi$. The radio core has not been Doppler de-boosted, so it is much more prominent than it would actually appear in the sky plane. In spite of the limitations, the right hand panel clarifies the intrinsic morphology of the radio source. Two diffuse structures exist to the north-east and south-west of the core, the radio lobes. This depiction helps one to visualize the lobes in the image on the left. We conclude that the source in the image in the left hand panel is likely a classical double lobe radio source that is being viewed near the jet axis. 


\section{1. $\bar{Q}$ Estimates from Archival Low Frequency Flux Density}

The estimate of $\bar{Q}$ in this paper relies on the methods of Rawlings and Saunders (1991); Willott et al. (1999); Blundell and Rawlings (2000), and will be referred to as the Oxford Method, hereafter. The estimator for $\bar{Q}$ was calibrated with a sample of 170 double lobe radio sources that were selected on the basis of low frequency emission $(151 \mathrm{MHz}-178 \mathrm{MHz})$. The calibrated estimator depends on only one parameter, a single value of flux density that is used as a surrogate for the flux density restricted to the radio lobes. This is the strength of the method, a single dish measurement at low frequency can be used to estimate $\bar{Q}$ for sources not in the calibration sample, as long as one can show that the measurement represents the lobe flux density. Thus, a single dish measurement is not applicable to blazars in general due to dilution of the lobe flux density with core and jet flux density.

The choice of $L_{151}$ as a surrogate for the luminosity of the radio lobes is motivated by the assumption that the core emission is attenuated by synchrotron self-absorption at $151 \mathrm{MHz}$ (Willott et al. 1999). We note that the sources in the calibration sample excluded known blazars since this would skew the calibration because there can be significant core and jet emission at $151 \mathrm{MHz}$ for a blazar. The energy stored in the lobes, $U$, can be estimated from $L_{151}$ and since the lobe plasma is low velocity this value will not be strongly affected by $\mathcal{D}$ (Willott et al. 1999). This is the sole function of the parameter $L_{151}$ in the Oxford Method. Consider the energy conservation equation (this is Equation (4) of Willott et al. (1999), EQW4, hereafter),

$$
\bar{Q}=\frac{f U_{\text {base }}\left(L_{151}\right)}{T},
$$

where $T$ is the source age, $U_{\text {base }}\left(L_{151}\right)$ is the minimum energy in the lobe assuming a low frequency cut off at $10 \mathrm{MHz}$, the jet axis is $60^{\circ}$ to the line of sight, there is no protonic contribution and $100 \%$ filling factor. ${ }^{1}$ The quantity $f$ incorporates deviations of actual radio lobes from these assumptions as well as energy lost expanding the lobe into the external medium, back flow from the head of the lobe and kinetic turbulence. Secondly, Equation (9) of Willott et al. (1999) relating $T$ and $\bar{Q}$ (EQW9, hereafter) is derived by considering the evolution of the lobe dimension, $R$, in models of lobe head advance into an ambient medium. Differentiating EQW9 yields an equation for $d R / d t$ (EQW10, hereafter) that is equated to

\footnotetext{
${ }^{1}$ Since we will ultimately be applying these formulae to blazars, the choice of the jet axis being $60^{\circ}$ to the line of sight needs some explanation. This is only used with regard to the calibration sample of double lobe radio sources. It is used to estimate the volume in the lobes It has nothing to do with the line of sight for which the formula can be applied. For example, double lobe quasars are believed to have a line of sight to the jet axis of $<45^{\circ}$ in the unified scheme for radio sources (Barthel 1989). Yet, the formula is routinely applied to quasars in the literature as it was intended.
} 
the lobe advance speeds derived from the distribution of length asymmetry between the two lobes of a sample of quasars in Scheuer (1995). EQW4, EQW9, EQW10 were solved simultaneously to eliminate $R$ and $T$, yielding $\bar{Q}$ as a function of $f$ and $L_{151}$ that is plotted in a scatter plot of the calibration sample in Figure 7 of Willott et al. (1999),

$$
\bar{Q} \approx 3.8 \times 10^{45} f L_{151}^{6 / 7} \mathrm{ergs} / \mathrm{s},
$$

The exponent on $f$ is 1 not $3 / 2$ as in Martinez-Sansigre and Rawlings (2011)

A more detailed effort to constrain $f$ that included gradients in the lobe magnetic field and significant deviations from equipartition estimated that $10<f<20$ (Blundell and Rawlings 2000). They assume a "relaxed" classical double lobe radio source in the derivation of their equations. Implicit in the relaxed constraint that was implemented in the determination of $f$ is that the linear size of the radio source is larger than the size of the host galaxy (i.e. $>20 \mathrm{kpc}$ ). Thus, the lobes are not confined by the pressure of the

relatively dense galactic medium. The assumed configuration is also implemented because a classical double lobe radio source is an object with a jet that has significant inclination to the line of sight (therefore small $\mathcal{D}$ ) that is dominated by two luminous lobes with a faint jet and core. In this configuration, $L_{151}$ is indeed an excellent surrogate for the lobe luminosity. We bound $\bar{Q}$ in Equations (2) from above (below) by using $f=20(f=10)$. Using $L_{151} \approx 4.5 \times 10^{28} \mathrm{WHz}^{-1} \mathrm{sr}^{-1}$ from Section 1 in Equation (1),

$$
\bar{Q}=1.65 \pm 0.55 \times 10^{47} \mathrm{erg} / \mathrm{s} .
$$

\subsection{Applying the Oxford Method to Blazars}

In Punsly and Tingay (2005), it was shown that one does not need a single dish measurement to estimate the lobe luminosity. A low frequency, high resolution, high sensitivity radio image can actually be used to determine the flux density in the lobes directly and this is equivalent to a single dish measurement in lobe dominated sources. More importantly, a high dynamic range image can be used to isolate the radio lobes from a blazar like radio core and jet, allowing a robust estimate of the flux density of the radio lobes. The additional data supplied by the radio image allows the Oxford Method to be applied to blazars. Recall that the sole purpose of $L_{151}$ in the derivation of Equation (2) was to estimate $U_{\text {base }}\left(L_{151}\right)$ in Equation (1). According to Equation (1) in Willott et al. (1999), the defining relation for $U_{\text {base }}\left(L_{151}\right)$, the minimum energy only depends on the combination $F_{\nu} \nu^{\alpha}$ which is a constant for a power law. Thus, it makes no difference if the one uses $2.5 \mathrm{GHz}$ or $151 \mathrm{MHz}$ to estimate $U$. Both choices have an uncertainty associated with $\alpha$ that is captured in the calibration of $f$. 
Unfortunately, due to the low declination, the maximum elevation above the horizon is low in New Mexico and PKS 0438-436 is visible for very short durations with the Very Large Array (VLA). The old VLA had insufficient u-v coverage to image the diffuse lobes during these brief observations. However, with the large bandwidths of the new Jansky VLA (JVLA), the $\mathrm{u}-\mathrm{v}$ coverage is much better and the sensitivity much higher. PKS 0438-436 was observed for 5-6 minutes throughout the summer of 2015 as part of Project $15 \mathrm{~A}-105$ as a phase calibrator. We utilize these observations in order to image the kpc structure of PKS 0438-436 for the first time and improve the $\bar{Q}$ estimate.

\subsubsection{Absolute Flux Calibration}

We used PKS 0438-436 not only as its own phase calibrator, but its own amplitude calibrator. In general this scheme seems flawed due to blazar variability. However, PKS 0438-436 is not that variable at lower frequency (eg., $2.5 \mathrm{GHz}$ ). Our frequency of observation is $2.5 \mathrm{GHz}$ in A-array. We found 18 ATCA (Australian Telescope Compact Array) observations from 1997 - 2011, from the ATCA calibrator web-page ${ }^{2}$ and Tingay et al. (2003); Massardi et al. (2008), all indicating a narrow range of flux density, $3-4 \mathrm{Jy}$.

We combine this steady nature of the flux with a fortuitous ATCA calibrator observation from $4.4 \mathrm{GHz}$ to $6.4 \mathrm{GHz}$ on August 13 2015. The extrapolated $2.5 \mathrm{GHz}$ flux density was $3.25 \pm 0.33$ Jy. There are JVLA observations on August 15 and August 24, 2015. The August 24 observation is imaged in Figure 1 since it had more uniform and symmetric coverage in the uv plane and this is the most important consideration for detecting diffuse, extended emission.

Those data were reduced using the EVLA pipeline incorporated in CASA version 4.7.2. As the data did not include a flux or bandpass calibrator, the pipeline turned out to be useful mostly for the editing (flagging) of bad data, which it successfully completed for all the 96 spectral windows (spw) and 64 channels. The corrected data were separated from the main dataset using the task SPLIT and imaged using CLEAN in the multi-frequency synthesis (mfs) widefield mode, with the number of Taylor coefficients (nterms) being 2 and number of w-projection planes being 128; "Briggs" weighting scheme was used for the imaging. One round of phase-only self-calibration was carried out before creating the final image. Finally, the AIPS verb RESCALE was used to correct the flux density scale. The core parameters using the AIPS Gaussian-fitting task JMFIT were: peak intensity $=2.74 \mathrm{Jy} \mathrm{beam}^{-1}$ and the integral intensity $=2.82 \mathrm{Jy}$ (see the left hand panel of Figure1). The extended flux density

\footnotetext{
${ }^{2}$ http://www.narrabri.atnf.csiro.au/calibrators/
} 
is $0.432 \pm 0.043 \mathrm{Jy}$, where the error is from the uncertainty in the amplitude calibrator.

\subsubsection{The Nature of the Radio Lobes and Jet Power}

We note that north-east and south-west extensions in emission were detected in 327 $\mathrm{MHz}$ Very Large Baseline Array (VLBA, hereafter) observations of Kanekar et al. (2009). These features appear to be a partially resolved jet and counter-jet on scales $\sim 100$ mas from the unresolved radio core. These smaller scale features are buried deep within the unresolved JVLA radio core (i.e., it is an order of magnitude smaller than the resolution of the $2.5 \mathrm{GHz}$ observations). The jet and counter-jet directions detected with the VLBA are consistent with jets that connect to the extended emission in Figure 1. This supports the notion that the partially resolved structures in the left hand panel of Figure 1 are distant radio lobes.

In Section 2.1, we discussed how the Oxford Method for estimating $\bar{Q}$ in Equation (2) is derived under the assumption of a relaxed classical double radio source. Thus motivated, we aim to establish that PKS 0438-436 is a classical double radio source except for the near polar line of sight. The elongated beam shape due to the proximity to the horizon makes the pole-on geometry difficult to interpret. The apparent separation of the radio lobes is about $50 \mathrm{kpc}$. However, this is not likely indicative of the actual physical separation of the lobes due to projection effects. Blazars that are high polarization quasars (HPQs), such as PKS 0438-436, have lines of sight (LOS) to the base of the jet that have been estimated from time variability brightness temperatures and the "inverse Compton catastrophe" to be $\approx 3.3^{\circ}$ (Hovatta et al. 2009). However, the jet is highly curved based on multi-frequency VLBA observations. The position angle of the jet direction rotates $\approx 90^{\circ}$ between 35 mas and 110 mas from the core (Kanekar et al. 2009; Tingay et al. 1998; Pushkarev and Kovalev 2012). Thus, one does not necessarily expect the same angle between the LOS and the jet at 110 mas and 2 arcsec from the core as the angle of the LOS to the jet base $(\ll 100$ mas from the point of origin). The $327 \mathrm{MHz}$ VLBA detection of a counter-jet to the south-west is inconsistent with the $\mathcal{D}$ associated with blazar activity. A possible explanation is the curving jet that results in a LOS, $\psi$, that is less aligned with the jet on scales $\sim 100$ mas and larger than that of an extreme blazar $\left(\approx 3.3^{\circ}\right)$, thereby reducing $\mathcal{D}$ (Lind and Blandford 1985). According to the unification scheme of FR II (Fanaroff Riley II) radio sources, the average line of sight to the $>10 \mathrm{kpc}$ jet in a quasar is $\psi \approx 30^{\circ}$ (Barthel 1989). The curved jet and counter-jet detection suggest a LOS larger than the HPQ value, $\psi>3.3^{\circ}$, but we still assume that it is more blazar-like than a typical radio loud quasar. Thus, we pick an intermediate value of $\psi \approx 15^{\circ}$ as a plausible value in order to qualitatively explore the intrinsic morphology of the radio source. 
Even if one assumes no interaction with the enveloping medium, comparing parameters of extended features such as lobes on the jet and counter-jet side of the core (i.e., flux or displacement from the core), one can determine at most the combination, $\beta_{\text {lobe }} \cos \psi$, for an individual object, where $\beta_{\text {lobe }}$ is the lobe advance speed (normalized to the speed of light) and $\psi$ is the angle of the line of sight to the lobe propagation direction (Scheuer 1995; Blundell and Rawlings 2000; Lind and Blandford 1985). The situation is exacerbated by a source without high signal to noise images of the radio lobes. First consider, the ratio of lobe separation on the jet (approaching) and counter-jet (receding) sides of the core $L_{\text {asym }}$. We approximate $L_{\text {asym }} \approx 1.05$ based on the radio image. Theoretically assuming intrinsic bilateral symmetry and no interaction with the enveloping medium, $L_{\text {asym }}=(1+$ $\left.\beta_{\text {lobe }} \cos \psi\right) /\left(1-\beta_{\text {lobe }} \cos \psi\right.$ ) (Scheuer 1995). The empirical estimated asymmetry of 1.05 and $\psi \approx 15^{\circ}$ implies that $\beta_{\text {lobe }} \approx 0.03$. Similarly, the ratio of flux density of the jetted lobe to counter-jetted lobe is estimated from the radio image as $F_{\text {asym }} \approx 1.8$. Theoretically, for a spherical region, intrinsic bilateral symmetry and minimal interaction with the enveloping medium, $\beta_{\text {lobe }} \cos \psi=\left[\left(F_{\text {asym }}\right)^{1 /(3+\alpha)}+1\right] /\left[\left(F_{\text {asym }}\right)^{1 /(3+\alpha)}-1\right]$ (Ribo et al. 2004). For a steep lobe spectral index of $\alpha=0.9$ (Kellermann et al. 1969), one finds for $F_{\text {asym }} \approx 1.8$ and $\psi \approx 15^{\circ}$ that $\beta_{\text {lobe }} \approx 0.08$. The values of $\beta_{\text {lobe }}=0.03$ and $\beta_{\text {lobe }}=0.08$, from $L_{\text {asym }}$ and $F_{\text {asym }}$, respectively agree with the findings of Scheuer (1995) of a most common value of $\beta_{\text {lobe }}=0.03 \pm 0.02$ and $\beta_{\text {lobe }}<0.15$ in FR II radio sources. This argument merely shows that $\psi \approx 15^{\circ}$ is consistent with what is known about lobe advance speeds, but it does not prove that this is a unique value. $\psi$ cannot be determined from the existing data. If $\psi$ were $\psi=10^{\circ}$ or $\psi=30^{\circ}$, the intrinsic length would be $\approx 350 \mathrm{kpc}$ or $\approx 100 \mathrm{kpc}$, respectively; yet the fundamental conclusion that the source is inherently a classical double lobe radio source being viewed along a LOS nearly parallel with the axis of the jet base is unchanged.

For illustrative purposes, we stretch the image so it appears as if the radio lobes have been rotated into the sky plane. This exercise is designed to elucidate the likelihood that PKS 0438-436 is inherently a classical double lobe radio source that happens to be viewed nearly pole-on to the base of the jet and therefore appears as a blazar within the standard unification scheme of FR II radio sources (Barthel 1989). The method of altering the image of PKS 0438-436, so that the lobes appear as they would if they were to lie in the sky plane instead of at $\psi \approx 15^{\circ}$ is fairly simple. The image in the left hand panel of Figure 1 is stretched by a factor of $1 / \sin 15^{\circ} \approx 4$ along the symmetry axis (the imaginary lines connecting the two lobes and the core) in Adobe Illustrator. The result is displayed in the right hand panel of Figure 1. This crude simulation does not correct for $\mathcal{D}$, so the core is much brighter than it would be if we were observing PKS 0438-436 in the sky plane. The implication of the figure (in spite of its limitations) is that PKS 0438-436 would be a classical double lobe radio sources if not for the fact that it is being viewed almost pole-on 
and it is being imaged with the added distortion of a very asymmetric beam. The choice of $\psi \approx 15^{\circ}$ does not affect this conclusion, only the intrinsic size of the object is affected by this choice. The stretched image highlights the existence of two lobes of extended emission that are approximately equidistant from the core and well outside of the galactic dimensions. These are the characteristics of a relaxed classical double radio source. Thus, the luminosity of these lobes are precisely the input that is intended to be used in the Oxford Method formula, Equation (2). The fact that we have not determined the exact intrinsic linear size or the actual value of $\psi$ is irrelevant to the intended use of the formula as discussed at length in Section 2.1

We assume that for the radio lobes $\alpha_{\text {lobe }}=0.9$ (Kellermann et al. 1969). Combining this with our estimate of the lobe flux density at $2.5 \mathrm{GHz}$ above and Equation (2),

$$
L_{151} \approx 3.0 \times 10^{28} \mathrm{WHz}^{-1} \mathrm{sr}^{-1}, \quad \overline{\mathrm{Q}}=1.14 \pm 0.38 \times 10^{47} \mathrm{erg} / \mathrm{s} .
$$

This seems to indicate that Equation (3) is an overestimate due to core and jet contributions to the $160 \mathrm{MHz}$ flux density. Equation (4) might be more accurate since the method of Willott et al. (1999) assumes that $L_{151}$ is a surrogate for the lobe luminosity. If $\alpha_{\text {lobe were }}$ 1.05 then the $7.9 \mathrm{Jy}$ single dish flux density at $160 \mathrm{MHz}$ could be attributed entirely to the lobes. However, this steep of a spectrum is an extremely rare circumstance (Kellermann et al. 1969).

\section{The Luminosity of the Accretion Flow}

In this section, we examine optical spectra in order to quantify the thermal luminosity of the accretion flow and the strength of the broad emission lines. Due to the high redshift, the UV emission line spectrum is redshifted into the optical band. The earlier spectrum (November 18 1977) in Figure 2 is from the RGO spectrograph on the $3.9 \mathrm{~m}$ Anglo Australian Telescope and this observation was used in the original determination of the quasar properties and redshift (Morton et al. 1978). The spectra from UVES, the Ultraviolet and Visual Echelle Spectrograph, on the VLT were retrieved from the ESO Spectral Data Products of Phase 3 archive portal. The UVES spectrum of Figure 2 results from the combination of eleven flux-calibrated spectra. On Sept. 14, 2002 a total of eight spectra were taken: four covering $3730 \AA-5000 \AA$ and four covering $6650 \AA$ - $10420 \AA$. On Oct. 30, 2003 three spectra covering $4720 \AA-6830 \AA$ were combined. A gap is present on the blue side of the redshifted CIV $\lambda 1549$, but the profile is presumably unaffected. There is also a small absolute flux mismatch around $6800 \AA$ between 2002 and 2003. The spectral resolution of UVES is about $\mathrm{R}=\lambda / \delta \lambda \sim 40,000$ with the slit opened to 1.008 ". We re-binned the spectra to a uniform scale $0.04 \AA /$ pixel in advance of the multi-component minimum $\chi^{2}$ best fitting analysis. 


\section{Optical Spectra of PKS 0438-436}
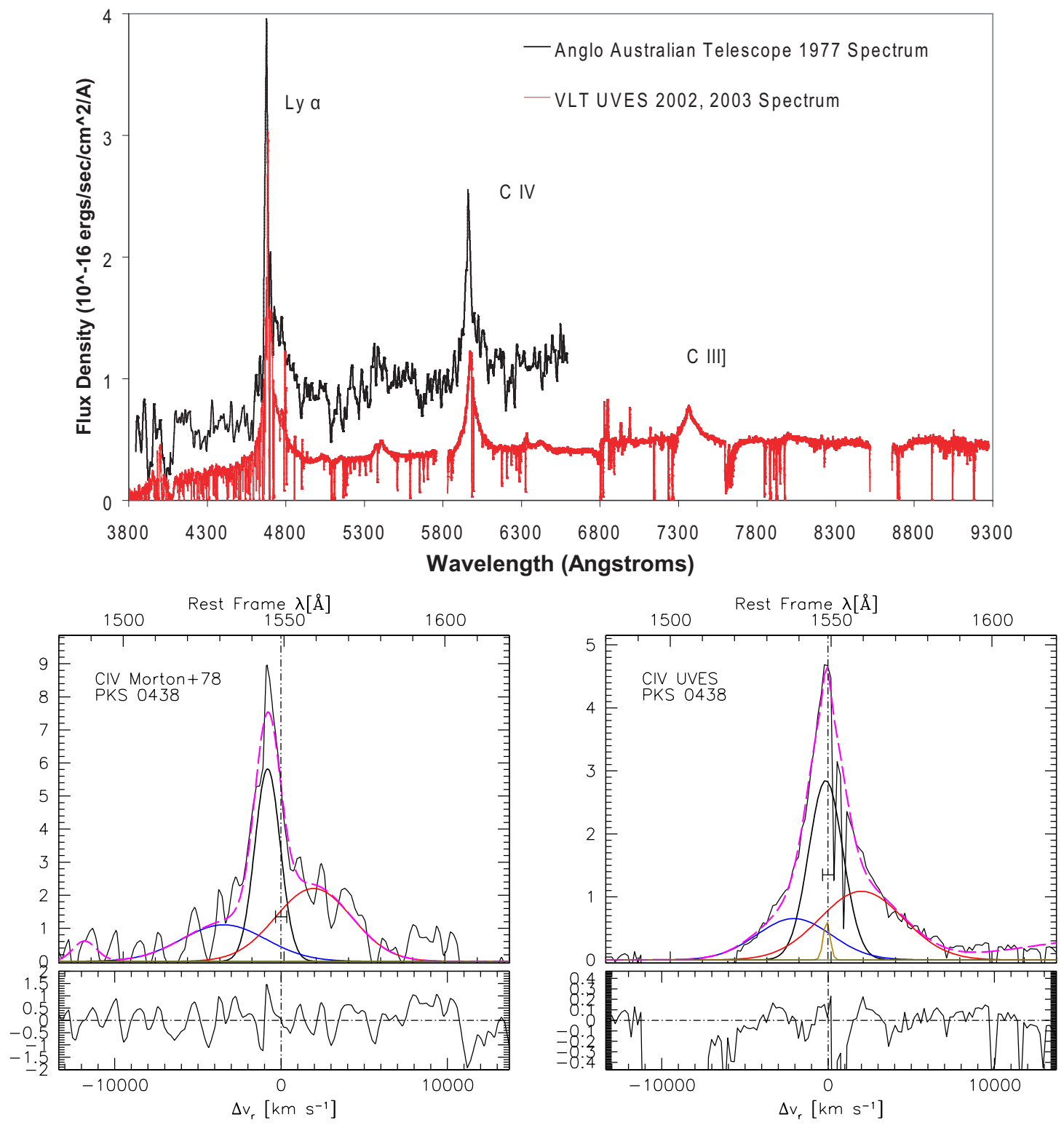

Fig. 2.- The top frame shows the two spectra. The bottom left panel is the CIV fit from the 1977 observation and the bottom right panel is the fit to the 2003 CIV. 
The luminosity appears larger in 1977 the black plot in the top frame of Figure 2). However, it should be noted that a significant flux correction was applied in Morton et al. (1978) to compensate for $\sim 3.5 "$ seeing and a 1.25" aperture. The rising spectrum with wavelength and higher flux level indicates that a strong optical synchrotron jet dominates the continuum. The spectral index is $\alpha_{\nu} \approx 3.4, F_{\nu} \sim \nu^{-\alpha_{\nu}}$ (Morton et al. 1978). Note that the plot in Figure 2 is with respect to wavelength. We confirm the Morton calculation. We estimate the flux density of the continuum as $F_{\lambda}(\lambda=6600 \AA) \approx 1.2 \times 10^{-16} \mathrm{ergs} / \mathrm{sec} / \mathrm{cm}^{2} / \AA$ and $F_{\lambda}(\lambda=4100 \AA) \approx 6.0 \times 10^{-17} \mathrm{ergs} / \mathrm{sec} / \mathrm{cm}^{2} / \AA$. Define the spectral index with respect to wavelength, $\alpha_{\lambda}$, as $F_{\lambda} \propto \lambda^{\alpha_{\lambda}}$. Then $\alpha_{\lambda} \approx \log (1.2 / 0.6) / \log (6600 / 4100) \approx 1.45 . \alpha_{\nu}=$ $2+\alpha_{\lambda} \approx 3.45$ in close agreement with the published result (Morton et al. 1978).

The emission line fits are presented in Table 1 and the CIV fits are shown in the bottom panels of Figure 2. The emission line fit was done simultaneously with the continuum power law fit. We used a $\chi^{2}$ minimization technique in IRAF with the task "specfit."

We decompose the broad emission lines (BELs) into three components, the BC (also called the intermediate broad line or IBL; Brotherton et al 1994), the redshifted BC (very broad component following Sulentic et al. 2000) and a blueshifted BC (Brotherton 1996; Marziani et al. 1996; Sulentic et al. 2000). The IBL/BC is represented by the black Gaussian profiles in Figure 2 and has been defined as a component with a FWHM $\sim 2000 \mathrm{~km} \mathrm{~s}^{-1}$ (Brotherton et al 1994). We occasionally add a weak narrow line that is at the systemic velocity of the quasar (brown). The BC needs some explanation in terms of the CIV decomposition. In Brotherton et al (1994), the BC was considered to have a blueshifted center frequency $(\mathrm{CF}) \sim 1000 \mathrm{~km} \mathrm{~s}^{-1}$ and a FWHM $\sim 7000 \mathrm{~km} \mathrm{~s}^{-1}$. According to Table 1 , there is a component like this in CIV, but it is less prominent than the red BC. The blueshifted BC has been found to be dominant in radio quiet quasars, but is less prominent and can be completely absent in radio loud quasars (Richards et al 2002; Punsly 2010). This was later interpreted in terms of Population B quasars in the classification scheme of (Sulentic et al. 2000). The Population B quasars include most of the radio loud quasars and typically have very large

Table 1: Broad Emission Line Fits

\begin{tabular}{|c|c|c|c|c|c|c|c|c|c|c|}
\hline Date & Line & $\begin{array}{l}\text { Red BC } \\
\text { CF } \\
\mathrm{km} \mathrm{s}^{-1} \\
\end{array}$ & $\begin{array}{l}\text { Red BC } \\
\text { FWHM } \\
\mathrm{km} \mathrm{s}^{-1} \\
\end{array}$ & $\begin{array}{c}\text { Red BC } \\
\text { Luminosity } \\
\text { ergs s }^{-1}\end{array}$ & $\begin{array}{l}\text { Blue BC } \\
\text { CF } \\
\mathrm{km} \mathrm{s}^{-1}\end{array}$ & $\begin{array}{c}\text { Blue BC } \\
\text { FWHM } \\
\mathrm{km} \mathrm{s}^{-1} \\
\end{array}$ & $\begin{array}{c}\text { Blue BC } \\
\text { Luminosity } \\
\text { ergs } \mathrm{s}^{-1}\end{array}$ & $\begin{array}{c}\text { IBL } \\
\text { FWHM } \\
\mathrm{km} \mathrm{s}^{-1} \\
\end{array}$ & $\begin{array}{c}\text { IBL } \\
\text { Luminosity } \\
\text { ergs s }^{-1}\end{array}$ & $\begin{array}{c}\text { Total BEL } \\
\text { Luminosity } \\
\text { ergs s }^{-1} \\
\end{array}$ \\
\hline $11 / 18 / 1977$ & Ly $\alpha$ & 2733 & $7143 \pm 136$ & $6.42 \times 10^{44}$ & -4558 & $3623 \pm 433$ & $8.20 \times 10^{43}$ & $1311 \pm 44$ & $3.91 \times 10^{44}$ & $1.11 \times 10^{45}$ \\
\hline $11 / 18 / 1977$ & CIV & 2823 & $5368 \pm 538$ & $3.11 \times 10^{44}$ & -3437 & $6026 \pm 903$ & $1.73 \times 10^{44}$ & $1806 \pm 75$ & $2.76 \times 10^{44}$ & $7.61 \times 10^{44}$ \\
\hline $9 / 14 / 2002$ & Ly $\alpha$ & 1422 & $6264 \pm 491$ & $3.16 \times 10^{44}$ & -1897 & $6410 \pm 785$ & $2.06 \times 10^{44}$ & $1747 \pm 52$ & $4.06 \times 10^{44}$ & $9.28 \times 10^{44}$ \\
\hline $10 / 30 / 2003$ & CIV & 2020 & $5865 \pm 312$ & $1.69 \times 10^{44}$ & -2063 & $5316 \pm 776$ & $9.10 \times 10^{43}$ & $2424 \pm 24$ & $1.82 \times 10^{44}$ & $4.41 \times 10^{44}$ \\
\hline $9 / 14 / 2002$ & $\mathrm{CIII}]$ & 2164 & $5855 \pm 183$ & $8.95 \times 10^{43}$ & $\mathrm{a}$ & $a$ & 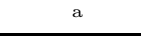 & $3256 \pm 221$ & $1.01 \times 10^{44}$ & $1.91 \times 10^{44}$ \\
\hline
\end{tabular}

${ }^{a}$ Not detected. 
black hole masses and low Eddington ratios: $R_{\mathrm{Edd}} \equiv L_{\mathrm{bol}} / L_{\mathrm{Edd}} \sim 0.01-0.1$, where $L_{\mathrm{bol}}$ is the thermal bolometric luminosity of the accretion flow and $L_{\mathrm{Edd}}=1.26\left(M_{b h} / M_{\odot}\right) \times 10^{38} \mathrm{ergs} \mathrm{s}^{-1}$ is the Eddington luminosity expressed in terms of the central supermassive black hole mass, $M_{b h}$ (Sulentic et al. 2007). The Population A quasars typically have smaller FWHM and higher Eddington ratios than Population B quasars (Sulentic et al. 2007). This decomposition of the quasar population is consistent with models of outwardly spiralling BEL gas that is driven by the intense radiation pressure form the accretion flow (Murray et al 1995). Population B CIV profiles do not tend to have a prominent blue excess at their base, but this excess in often detectable. In this respect, PKS 0438-436 seems to have properties of CIV which are consistent with the radio loud Population B quasars in terms of the distribution of centroid line shifts (Sulentic et al. 2007). Empirically, the blue BC might represent the existence of a moderate $R_{\mathrm{Edd}}$ accretion flow, and the even stronger red $\mathrm{BC}$ is correlated with the extremely powerful jet (Punsly 2010).

We wish to estimate $L_{\mathrm{bol}}$ in a manner that does not include reprocessed radiation in the infrared from distant molecular clouds. This would be double counting the thermal accretion emission that is reprocessed at mid-latitudes (Davis and Laor 2011). The accretion flow continuum may be hidden by strong synchrotron dilution. As is commonly done for blazars, we use the BEL luminosity, $L(C I V)$, from Table 1 as a surrogate for $L_{\mathrm{bol}}$ (Wang et al. 2004). We employ an estimator obtained by comparing $L(C I V)$ to the Hubble Space Telescope ultraviolet composite continuum for quasars with $L_{\mathrm{bol}} \gtrsim 10^{46} \mathrm{ergs} / \mathrm{sec}$, (Zheng et al. 1997; Telfer et al. 2002; Punsly et al. 2016)

$$
\begin{aligned}
& L_{\mathrm{bol}}=(107 \pm 22) L(C I V) \approx \\
& 4.7 \pm 1.0 \times 10^{46} \mathrm{ergs} / \mathrm{s}: \text { October } 2003, \quad 8.1 \pm 1.7 \times 10^{46} \mathrm{ergs} / \mathrm{s}: \text { November } 1977 .(5)
\end{aligned}
$$

The uncertainty in Equation (5) is the uncertainty in the Hubble Space Telescope composite continuum level and the uncertainty in $L(C I V$ ) added in quadrature (Telfer et al. 2002). We can compare this to an estimate from the low state continuum in the far UV in order to see how much the steep synchrotron tail dominates in this region of the spectrum. From the spectrum in Figure 2 and the formula expressed in terms of quasar cosmological rest frame wavelength, $\lambda_{e}$ and spectral luminosity, $L_{\lambda_{e}}$, from Punsly et al. (2016),

$$
L_{\mathrm{bol}} \approx(4.0 \pm 0.7) \lambda_{e} L_{\lambda_{e}}\left(\lambda_{e}=1350 \AA\right) \approx 4.5 \pm 0.8 \times 10^{46} \mathrm{ergs} / \mathrm{s} .
$$

This estimate from September 2002 is similar to our lower BEL estimate in Equation (5) from 2003. Thus, just longward of Ly $\alpha$ it appears that the accretion disk continuum is dominating over the synchrotron component. Combining Equations (3) - (6) we find that $\bar{Q} / L_{\mathrm{bol}}=3.3 \pm 2.6$ which is extremely high for a quasar (Punsly 2010). 


\section{The Gamma Ray Flare}

A preliminary FERMI detection of PKS 0438-436 on December 112016 was announced in ATel 9854, it previously had no gamma ray detections; EGRET, AGILE or FERMI (Cheung 2016). We have performed the data analysis of Fermi-LAT data using the fermipy framework Wood et al. (2017) version 0.16.0, based on the Fermi Science Tools $v 11 r 5 p 3$ and P8R2_SOURCE_V6 instrument response functions. The data have been selected in the time span of six days, between 57731.0 MJD 57737.0 MJD. We have selected photons from a region of interest (ROI) with a radius of $10^{\circ}$, centered on PKS 0438-436, and we have built a model of the ROI using sources within $15^{\circ}$ distance from the ROI center and reported in the FermiLAT Third Source Catalog (3FGL) Acero et al. (2015), including both the emission from the Galactic diffuse (gll_iem_v06.fits) and the isotropic iso_P8R2_SOURCE_V6_v06.txt backgrounds.

For the light curve extraction we have used bins of 18 hours, for a time range starting on MJD 57731.00 and ending on MJD 57736.25. For each bin a binned likelihood analysis is performed, using la power-law function, and freeing all the sources located within a distance of $5^{\circ}$ from the ROI center. The light curve is shown in Figure 3, top frame. The top left frame reports the integrated photon flux in the $100 \mathrm{MeV}-100 \mathrm{GeV}$ energy range, and the top right frame reports the corresponding apparent luminosity. The source is detected in four bins with a significance $>3 \sigma$, and the flaring activity in concentrated during a time span of 54 hours centered on MJD 57734.4.

We have performed a time resolved spectral analysis for each flaring bin in the 54-hours time span, and for the full 54-hours time span, using a power-law model $\left(d N / d E \propto E^{-\alpha_{\gamma}}\right)$, and freeing all the sources located within a distance of $5^{\circ}$ from the ROI center. The results are summarized in Table 2 . There are 6 sources within the $5^{\circ}$ region, and all these sources show, during the flare period, a flux level at least a factor of 10 times smaller than the flux of PKS 0438-436. The source is detected with a significance of $\gtrsim 10 \sigma$ during the full flare period, and with a significance ranging from $\gtrsim 3 \sigma$ to $\gtrsim 8 \sigma$, during each time bin. There is formally no detection above $10 \mathrm{GeV}$, only upper limits. The lack of detected photons arises from

Table 2: Fermi-LAT Spectral Analysis Summary

\begin{tabular}{|c|c|c|c|c|c|c|c|c|}
\hline Time range & start & stop & $\begin{array}{l}\operatorname{delta} \mathrm{T} \\
\text { hours }\end{array}$ & $\overline{\alpha \gamma}$ & $\begin{array}{c}\text { Number } \\
\text { of photons } \\
\text { detected }\end{array}$ & $\begin{array}{c}\sqrt{\mathrm{TS}} \\
\text { statistical } \\
\text { significance }\end{array}$ & $\begin{array}{l}\text { Luminosity } \\
10^{49} \text { ergs s }^{-1}\end{array}$ & Truncation Energy \\
\hline flaring & 57733.25 & 57735.50 & 54 & $2.2 \pm 0.1$ & 78 & 10.90 & $4.8 \pm 1.0$ & $100 \mathrm{GeV}$ \\
\hline $\mathrm{a}$ & 57733.25 & 57734.00 & 18 & $2.2 \pm 0.2$ & 41 & 8.30 & $5.8 \pm 1.6$ & $100 \mathrm{GeV}$ \\
\hline b & 57734.00 & 57734.75 & 18 & $2.4 \pm 0.4$ & 14 & 3.38 & $2.2 \pm 1.1$ & $100 \mathrm{GeV}$ \\
\hline c & 57734.75 & 57735.50 & 18 & $2.0 \pm 0.2$ & 22 & 6.65 & $6.6 \pm 3.0$ & $100 \mathrm{GeV}$ \\
\hline flaring & 57733.25 & 57735.50 & 54 & $2.2 \pm 0.1$ & 77 & 10.88 & $4.1 \pm 0.6$ & $10 \mathrm{GeV}$ \\
\hline $\mathrm{a}$ & 57733.25 & 57734.00 & 18 & $2.2 \pm 0.2$ & 41 & 8.32 & $5.0 \pm 1.1$ & $10 \mathrm{GeV}$ \\
\hline b & 57734.00 & 57734.75 & 18 & $2.4 \pm 0.5$ & 13 & 3.30 & $2.0 \pm 0.9$ & $10 \mathrm{GeV}$ \\
\hline c & 57734.75 & 57735.50 & 18 & $1.9 \pm 0.3$ & 21 & 6.57 & $4.9 \pm 1.6$ & $10 \mathrm{GeV}$ \\
\hline
\end{tabular}



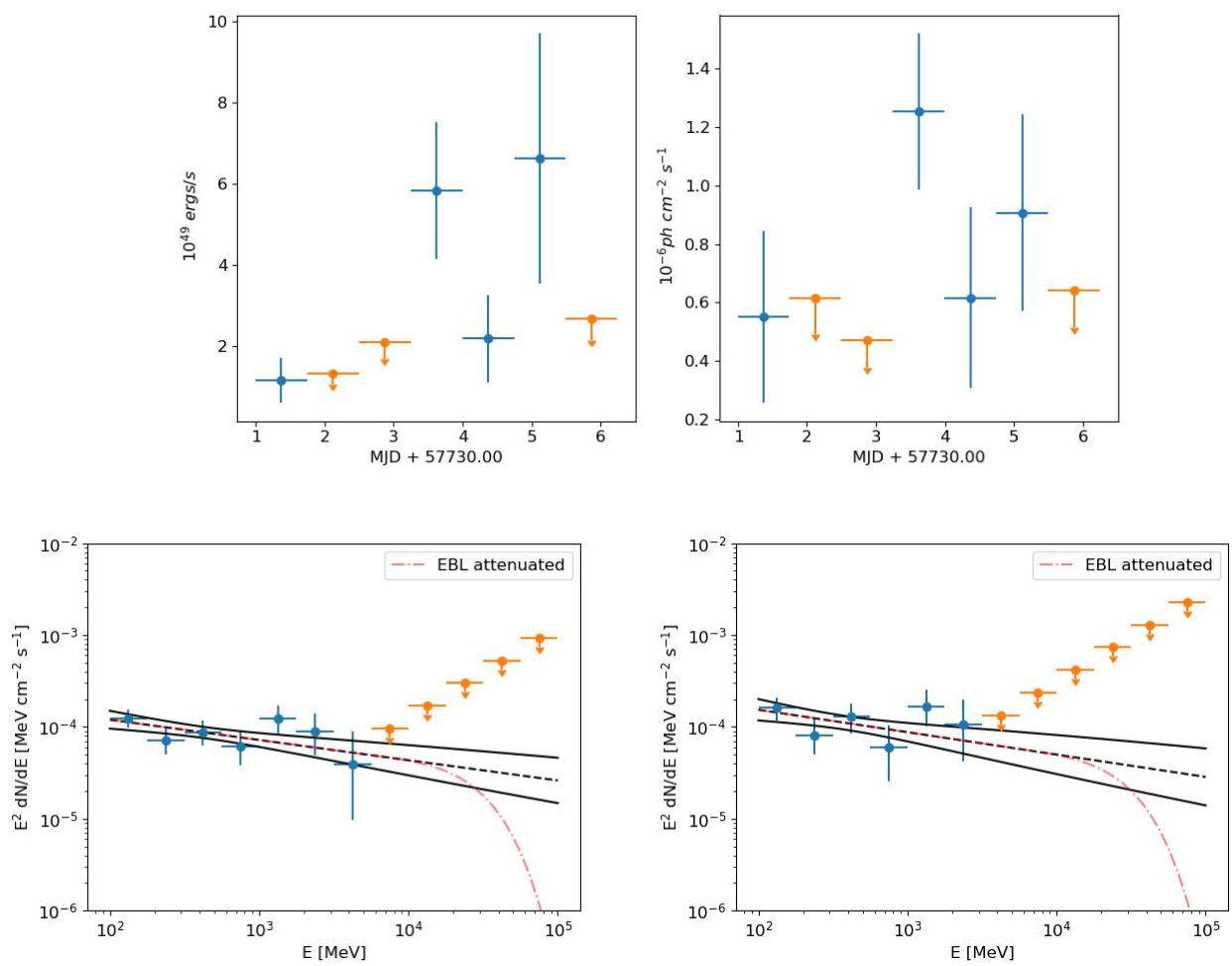

SED PKS 0438-436

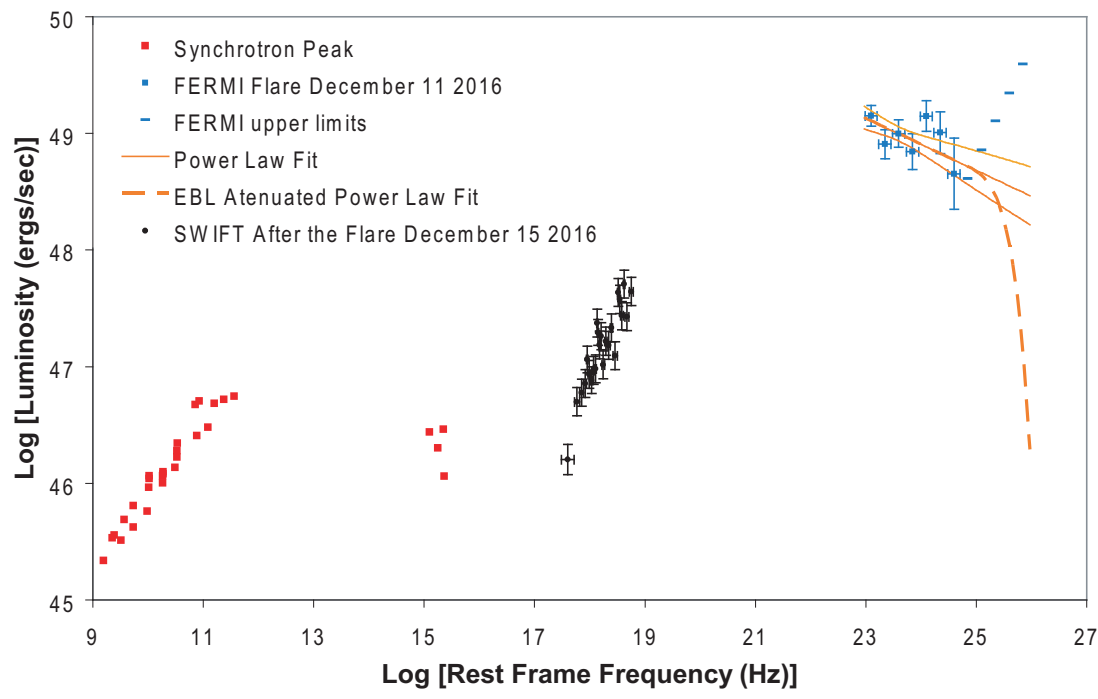

Fig. 3.- The top frame is the $100 \mathrm{MeV}-100 \mathrm{GeV}$ light curves in terms of apparent luminosity and count rate. The middle frames are the spectral fits averaged over the 54 hours of the flare (left), and the $a$ time range ( right). The red dot-dashed line corresponds to the best fit model attenuated by the EBL, see the text for more details. The bottom frame is an SED (not contemporaneous) from the radio band to $\gamma$-rays. 
two conspiring issues. First is the large distance that makes for low number statistics even for this very luminous flare. Second is the EBL (extra background light) opacity. Gamma ray extinction occurs as a consequence of pair creation in the soft background photon field (Gould and Schreder 1967; Franceschini et al. 2008). In order to understand the impact of the EBL on our data, we have calculated the EBL attenuation using the $\gamma-\gamma$ optical depths evaluated at $\mathrm{z}=2.85$ from the template model in Finke (2010). ${ }^{3}$ The red dot-dashed line in the bottom frames of Figure 3 shows the expected attenuation. The EBL starts to be relevant above $\approx 70 \mathrm{GeV}$, and the upper limits in our SEDs starts at $\approx 10 \mathrm{GeV}$. For this reason we were unable to disentangle the EBL extinction from the intrinsic high energy cutoff. The only intrinsic fit warranted by the data is a power law. Table 2, presents power law fits that are truncated at $10 \mathrm{GeV}$ and $100 \mathrm{GeV}$.

The SED in Figure 3 are radio data from the NASA Extragalactic Database, optical from Figure 2, and the FERMI fit to the 54 hours of December 112016 (time range flaring in Table 2). We also plot the SWIFT spectrum, observed two days after the flare, obtained using the Swift XRT products generator ${ }^{4}$ (Evans et al. 2009). The X-ray spectrum is more luminous and harder than historical X-ray spectra (Cappi et al. 1997; Reeves and Turner 2000; Brocksopp et al. 2004). The data are not contemporaneous, but the strong inverse Compton (IC) $\gamma$-ray peak $>5 \times 10^{49} \mathrm{ergs} \mathrm{s}^{-1}$ is much stronger than the synchrotron peak $\sim 2 \times 10^{47} \mathrm{ergs} \mathrm{s}^{-1}$, characteristic of strong quasar gamma ray sources (Ghisellini et al. 2010). The luminosity of the flare in Table 2 is $\sim 100$ times the quiescent upper limit (August 4 2008 - July 4 2009) in Böck et al. (2016) indicating extreme variability. Since it is rarely detected, the time averaged $\gamma$-ray luminosity is not extreme (Böck et al. 2016). However, the flare is $\approx 55-65 \%$ as luminous (in an 18 hour window) as the historically large flare of 3C 454.3 even though the peak of the SED is redshifted out of the FERMI observing window (Abdo et al. 2011).

Considering $\mathcal{D}$, it is of interest to estimate the intrinsic luminosity of the flare. For an unresolved source $\mathcal{D}=\delta^{3+\alpha}$, where $\delta$ is the Doppler factor (Lind and Blandford 1985). Based on Table $2 \alpha \equiv \alpha_{\gamma}-1 \approx 1.2$. Thus, $\mathcal{D} \approx 15^{4} \approx 5 \times 10^{4}$, using the average of $\delta=15$ for high optical polarization quasars estimated from time variability (Hovatta et al. 2009). This implies an intrinsic luminosity of the flare $\approx 10^{45} \mathrm{ergs} / \mathrm{s} \sim 0.01 \overline{\mathrm{Q}}$. These radiation losses are easily sustainable by a jet with a power similar to the long term time average.

\footnotetext{
${ }^{3}$ http://www . phy . ohiou.edu/ finke/EBL/index.html

${ }^{4}$ http: //www.swift.ac.uk/user\_objects/
} 


\section{Conclusion}

This Letter is the the first comprehensive description of PKS 0438-436.. In Section 2, we provided a JVLA image of the radio source that delineates the kpc structure for the first time. In Equations (3)-(4) we estimate $\bar{Q}=1.48 \pm 0.72 \times 10^{47} \mathrm{erg} \mathrm{s}^{-1}$ near the maximum value known for quasars (Willott et al. 1999). In section 3, we describe the optical spectrum. We estimate $L_{\text {bol }}$ from the CIV BEL in two different epochs: $L_{\text {bol }}=4.7 \pm 1.0 \times 10^{46} \mathrm{ergs} / \mathrm{s}$ : October $2003, \mathrm{~L}_{\mathrm{bol}}=8.1 \pm 1.7 \times 10^{46} \mathrm{ergs} / \mathrm{s}:$ November 1977 . We also estimate $L_{\mathrm{bol}}$ from the far UV continuum in a low state in September 2002: $L_{\text {bol }}=4.5 \pm 0.8 \times 10^{46} \mathrm{ergs} / \mathrm{s}$. There is agreement with the BEL estimate a year later. This indicates the extremely large normalized

jet power, $\bar{Q} / L_{\text {bol }}=3.3 \pm 2.6$, amongst the most extreme known for quasars (Punsly 2007). In spite of this, PKS 0438-436 has only been detected once in the $\gamma$-rays. This is likely an extreme manifestation of external Compton scattering in a relativistic jet for which the apparent luminosity is very sensitive to the geometry (Dermer and Schlickeiser 1993).

This work was partially based on observations collected at the European Organisation for Astronomical Research in the Southern Hemisphere under ESO programme(s) 69.A0051(A) and 072.A-0346(A). Based on data obtained from the ESO Science Archive Facility under request number 295401. This work was supported by the National Radio Astronomy Observatory, a facility of the National Science Foundation operated under cooperative agreement by Associated Universities, Inc., Project 15A-105. Partial funding for this work was provided by ICRANet.

\section{REFERENCES}

Abdo, A. A., Ackermann, M., Ajello, M., et al. 2011, ApJL, 733, 26

Acero, F., Ackermann, M., Ajello, M., et al. 2015,ApJS, 218, 23, doi: 10.1088/0067$0049 / 218 / 2 / 23$

Barthel, P. D. 1989, ApJ, 336, 606

Blundell, K., Rawlings, S. 2000 AJ 1191111

Böck, M.; Kadler, M.; Mller, C.; Tosti, G.; Ojha, R.; et al. 2013 A \& A 59040

Brocksopp C., Puchnarewicz E. M., Mason K. O.,Cordova F. A., Priedhorsky W. C., 2004, MNRAS, 349, 687

Brotherton, M., Wills B., Steidel, C., Sargent, W. 1994, ApJ 430131 
Brotherton, M. 1996, ApJS 1021

Cappi M., Matsuoka M., Comastri A., Brinkmann W., Elvis M., Palumbo G. G. C., Vignali C., 1997, ApJ, 478, 492

Cheung, C. 2016, ATel 9854 http://www.astronomerstelegram.org/?read=9854

Davis, S., Laor, A. 2011, ApJ 72898

Dermer C. D., Schlickeiser R., 1993, ApJ, 416, 458

Evans P. A., et al., 2009, MNRAS, 397, 1177

Finke, J. D., Razzaque, S., \& Dermer, C. D. 2010, The Astrophysical Journal, 712, 238

Franceschini, A.; Rodighiero, G.; Vaccari, M. 2008, A \& A, 487837

Fugmann, W.; Meisenheimer,K. 1988, A\&AS, 76145

Ghisellini, G, Tavecchio, F. and Foschini, L. et al. 2010 MNRAS 402497

Gould R. J., Schreder G., 1967, Physical Review, 155, 1404

Hovatta, T.; Valtaoja, E.; Tornikoski, M.; Lhteenmki, A. 2009, A \& A 498, 723

Impey, C. and Tapia, S., 1990 ApJ 354124

Kanekar, N.; Lane, W. M.; Momjian, E.; Briggs, F. H.; Chengalur, J. N. 2009, MNRAS Lett, 394, 61

Kellermann, K. I., Pauliny-Toth, I. I. K., Williams, P. J. S. 1969 ApJ 1571

Lind, K., Blandford, R.1985 ApJ 295358

Martinez-Sansigre, A. and Rawlings S. 2011, MNRAS Lett. 41884

Marziani, P., Sulentic, J., Dultzin-Hacyan, D., Calvani, M., Moles, M. 1996, ApJS 10437

Marziani, P., Sulentic, J., Stirpe, G., Zamfir, S., Calvani, M. 2009, A\&A 49583

Massardi, M., Ekers, R., Murphy, T., et al. 2008, MNRAS 384775

Morton, D. C.; Savage, A.; Bolton, J. G. 1978, MNRAS 185735

Murray, N. et al 1995, ApJ 451498

Punsly, B. 1995 AJ 1091555 
Punsly, B. and Tingay, S. 2005 ApJL 63389

Punsly, B. 2007, MNRAS, 381, L79

Punsly, B. 2010, ApJ 713232

Punsly, B., Marziani, P., Zhang, S., Muzahid, S., O’Dea, C. 2016, ApJ 830, 104

Pushkarev, A. and Kovalev., Y. 2012 A\&A 54434

Rawlings S., Saunders R., 1991, Nat, 349, 138

Reeves J. N., Turner M. J. L., 2000, MNRAS, 316, 234

Richards, G. et al 2002, AJ 1241

Reuter, H.-P., Kramer, C., Sievers, A., et al. 1997, A \& ASS, 122, 271

Ribo, M., Dhawan, V. Mirabel, F. 2004, Proc. of the 7th VLBI Network Symposium, Bachiller, R., Colomer, F., Desmurs, J., de Vicenete, P. (eds) Toledo, Spain astro$\mathrm{ph} / 0412657$

Scheuer P. A. G., 1995, MNRAS, 277, 331

Slee, O. 1995, AuPh 48143

Sulentic, J., Marziani, P., and Dultzin-Hacyan, D. 2000 ARA\& A 38, 521

Sulentic, J., Bachev, R., Marziani,P., Negrete, C. A., Dultzin, D. 20007 ApJ 666757

Telfer, R., Zheng, W., Kriss, G., Davidsen, A. 2002, ApJ 565773

Tingay, S. J.; Murphy, D. W.; Lovell, J. E. J. et al. 1998, ApJ, 497, 594

Tingay S. J., Jauncey D. L., King E. A., Tzioumis A. K., Lovell J. E. J., Edwards P. G., 2003, PASJ, 55, 351

Wang, J.-M., Luo, B, Ho, L. 2004 ApJL 6159

Willott, C., Rawlings, S., Blundell, K., Lacy, M. 1999, MNRAS 3091017

Wood, M., Caputo R., Charles E., Di Mauro, M., Magill, Perkins J., for the Fermi LAT Collaboration, arXiv:1707.09551

Zheng, W. et al 1997, ApJ 475469 